\title{
A 15q13.3 microdeletion segregating with autism
}

\author{
Alistair T Pagnamenta ${ }^{1}$, Kirsty Wing ${ }^{1}$, Elham Sadighi Akha ${ }^{1,2}$, Samantha JL Knight ${ }^{1,2}$, \\ Sven Bölte ${ }^{3}$, Gabriele Schmötzer $^{3}$, Eftichia Duketis ${ }^{3}$, Fritz Poustka ${ }^{3}$, Sabine M Klauck ${ }^{4}$, \\ Annemarie Poustka ${ }^{4}$, Jiannis Ragoussis ${ }^{1}$, Anthony J Bailey ${ }^{5}$, Anthony P Monaco ${ }^{* 1}$ and the \\ International Molecular Genetic Study of Autism Consortium (IMGSAC) ${ }^{6}$
}

\author{
${ }^{1}$ The Wellcome Trust Centre for Human Genetics, University of Oxford, Oxford, UK; ${ }^{2}$ The Oxford Partnership \\ Comprehensive Biomedical Research Centre, The Wellcome Trust Centre for Human Genetics, University of Oxford, \\ Oxford, UK; ${ }^{3}$ Klinik für Psychiatrie und Psychotherapie des Kindes-und Jugendalters, JW Goethe Universität, Frankfurt, \\ Germany; ${ }^{4}$ Abteilung Molekulare Genomanalyse, Deutsches Krebsforschungszentrum, Heidelberg, Germany; \\ ${ }^{5}$ University Department of Psychiatry, Warneford Hospital, Oxford, UK
}

Autism and mental retardation (MR) show high rates of comorbidity and potentially share genetic risk factors. In this study, a rare $\sim 2 \mathrm{Mb}$ microdeletion involving chromosome band $15 \mathrm{q} 13.3$ was detected in a multiplex autism family. This genomic loss lies between distal break points of the Prader-Willi/Angelman syndrome locus and was first described in association with MR and epilepsy. Together with recent studies that have also implicated this genomic imbalance in schizophrenia, our data indicate that this CNV shows considerable phenotypic variability. Further studies should aim to characterise the precise phenotypic range of this CNV and may lead to the discovery of genetic or environmental modifiers.

European Journal of Human Genetics (2009) 17, 687-692; doi:10.1038/ejhg.2008.228; published online 3 December 2008

Keywords: autism; CNV; genetic modifier; learning disability; schizophrenia; phenotypic variability

\section{Introduction}

Twin studies have consistently indicated a strong genetic susceptibility to autism. ${ }^{1}$ Analysis of chromosomal aberrations and genetic linkage studies have helped identify numerous autism susceptibility regions at many different chromosomal loci. ${ }^{2,3}$ One of the first regions to be implicated was the imprinted Prader-Willi/Angelman syndrome (PW/AS) region on the long arm of chromosome 15. ${ }^{4}$ Duplications at this locus are found to be associated with autism only when inheritance of this duplication is maternal or when de novo events originate on the maternal chromosome. ${ }^{5}$ The latest whole-genome CNV studies confirm that the 15q11-13 duplication is found in about

*Correspondence: Professor AP Monaco, Wellcome Trust Centre for Human Genetics, University of Oxford, Roosevelt Drive, Headington, Oxford OX3 7BN, UK.

Tel: + 441865 287502; Fax: + 441865 287650;

E-mail: anthony.monaco@well.ox.ac.uk

${ }^{6}$ See http://www.well.ox.ac.uk/monaco/autism/IMGSAC.shtml

Received 12 September 2008; revised 30 October 2008; accepted 31 October 2008; published online 3 December 2008
$0.5-0.7 \%$ of individuals with autism, ${ }^{6,7}$ although this is likely to be an underestimate as autism cohorts are often pre-screened by karyotyping.

A recent study by Sharp et $a l^{8}$ identified a recurrent microdeletion around the distal PW/AS break points in approximately $0.3 \%$ of individuals with mental retardation (MR), with the region between break points BP4 and BP5 considered to be critical. One of the nine cases described in this study presented with mild autistic traits. Learning disability and autism often show high levels of comorbidity, with MR typically observed in up to $70 \%$ of individuals with autism. ${ }^{9}$ MR and autism are also thought to share genetic risk factors. A recent study suggested that as many as two-thirds of male patients carrying the full Fragile $\mathrm{X}$ expansion (>200 CGG repeats) exhibit autistic traits. ${ }^{10}$ Similarly, the recurrent $16 \mathrm{p} 11.2$ microdeletion/duplication has been associated with autism and psychiatric disorders ${ }^{7}$ and was linked earlier to mild MR, with seizures and abnormalities of the aortic valve. ${ }^{11}$

The findings described above led us to investigate further the segregation of a microdeletion of $15 q 13.3$, similar to 
the critical region involved in MR, identified in a family in which three boys were all affected with autism. The results of these studies, together with detailed clinical information, are presented and the genotype-phenotype correlation in this family is compared with those in the individuals described by Sharp et al. ${ }^{8}$

\section{Methods and results \\ Case reports}

Case 1 The birth of the proband was induced due to a deterioration in foetal heart rate, following premature rupture of membranes. He spent the first week of his life in an incubator and suffered from prolonged neonatal jaundice. Language development was markedly delayed with first words at 4 years and phrases at 6 years. Articulation, although severely affected initially, was good at the time of referral. He demonstrates severe obsessional and hyperactive behaviours, has very limited social communication and poor eye contact. A neurological examination indicated slightly disturbed coordination and dysdiadochokinesia.

Case 2 The second child (half-sib of the proband) was born after a normal pregnancy with no complications. He spent a week in hospital, aged 15 months, due to severe enteritis. He showed delayed language development with first words at 3 years and phrases at 4 years. Aged 3 years, he was socially withdrawn, with hyperactive behaviour, and was unable to join in with games. At a later examination, it was noted that he showed irritable behaviour in groups, often living in his own world and refusing eye contact. He developed obsessional interests and showed various ritualistic behaviours, such as washing.

Case 3 The youngest child (half-sib of the proband, full-sib of case 2) was born by a caesarean section (to avoid breech birth) after a normal pregnancy. Language development was markedly delayed with first words at 5 years 5 months and phrases at 6 years. The mother noticed that from 6 months he was socially withdrawn, never joining in with activities or developing emotional relationships. He was fixated with certain objects and still sometimes used idiosyncratic speech with articulation abnormalities. A neurological examination showed muscular hypotonia, disturbed coordination, dysdiadochokinesia and no dominant laterality.

All three boys received a clinical diagnosis (ICD-10) of childhood autism. They also met the Autism Diagnostic Interview-Revised (ADI-R) algorithm criteria for autism and the autism spectrum criteria using the Autism Diagnostic Observation Schedule (ADOS) (case 3 has narrowly defined autism on both assessments). All three children had a history of language delay and full-scale IQs ranged from 72

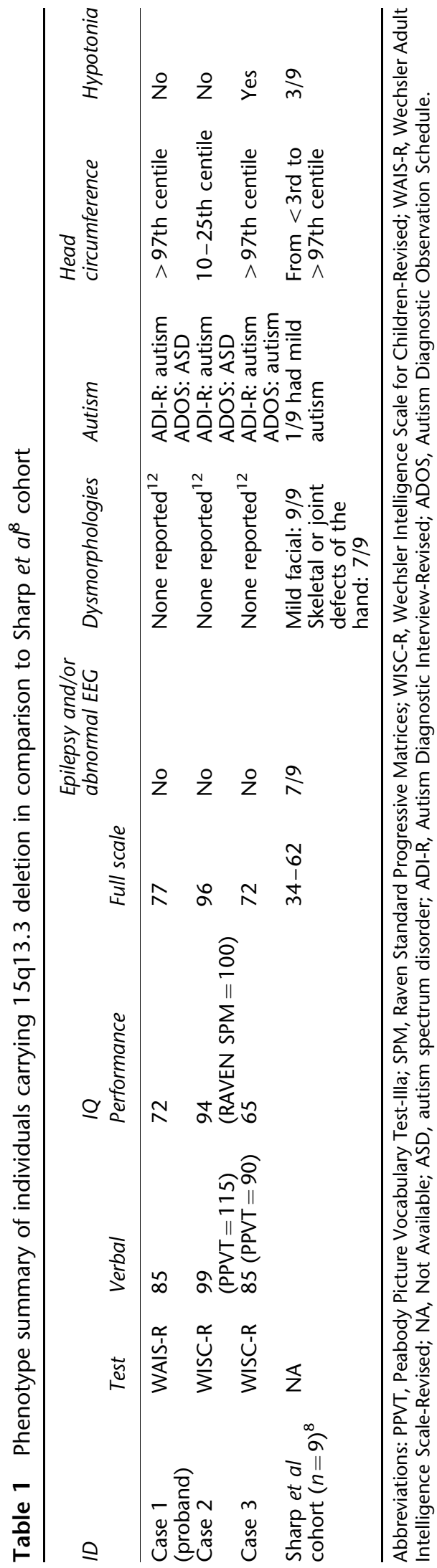


to 96 (Table 1) using the revised Wechsler Intelligence Scales for Children or Adults. The family was of Caucasian ethnicity and had been shown to be negative for Fragile $\mathrm{X}$ and karyotype (G-banding) abnormalities. Neurological and physical examination was carried out on all three cases using a standardised protocol ${ }^{12}$ and no obvious dysmorphologies such as hypertelorism or fifth finger clinodactyly were seen; however, we cannot rule out the presence of more subtle changes. More detailed clinical information is presented in Table 1 to allow comparison with cases described by Sharp et al. ${ }^{8}$

Both psychologists involved with the clinical assessment of this family deemed the mother to be of good intellectual capacity, with no suggestion of any psychopathology.

\section{Molecular genetics}

DNA from this family was collected over 13 years ago and has already been used in several genetic studies. ${ }^{13,14}$ Consequently, only whole-genome amplified (WGA) material (REPLI-g, QIAGEN) was available for an Autism Genome Project (AGP) study using the Human $1 \mathrm{M}$ BeadChip SNP array (Illumina, San Diego, CA, USA). Following genome-wide $\mathrm{CNV}$ analysis of this SNP data using QuantiSNP ${ }^{15}$ (with $\mathrm{L}=1 \mathrm{M}, \mathrm{EM}$ iterations $=25$ and using the GC correction option), a high level of false positives were seen in these samples: 1478, 1757 and 1779 CNVs were detected with log Bayes Factor $>10$ for the proband, his mother and his father respectively, compared with a mean of $\sim 50$ in genomic DNA samples. This led to the exclusion of this family from the main AGP CNV analysis (in progress). A recent study has similarly shown that SNP-based CNV analysis gives high levels of false positives for WGA samples, but suggests that, after comparison with similarly amplified control samples, CNVs with the highest significance values may be real. ${ }^{16}$ In this family, the CNV detected with most significance was a loss spanning the PW/AS BP4 to BP5 region (Figure 1), identified in the proband (chr15: 28736917-30686830, b36 genome coordinates, $\log \mathrm{BF}=550)$ and apparently being transmitted from the mother (chr15: $28736917-$ $30303265, \log B F=574$ ). Despite the uncertainty of these findings, the fact that this putative loss closely matched with the recently described $15 \mathrm{q} 13.3$ recurrent MR microdeletion $^{8}$ and was unlikely to be a WGA-specific artefact (it was not present in the father or other WGA samples) led us conclude that it may be a true finding worth validating. The microdeletion was subsequently verified using arraybased comparative genomic hybridisation (aCGH). Briefly, new aliquots of whole-blood genomic DNA were obtained and tested against a genome-wide $44 \mathrm{~K}$ oligonucleotide aCGH platform (Agilent Technologies, Santa Clara, CA, USA) according to the manufacturer's instructions, using well-characterised, sex-matched control DNA as the reference. The aCGH analysis verified the genomic loss, with a minimal deleted region of chr15: 28 725 507-30 701432.
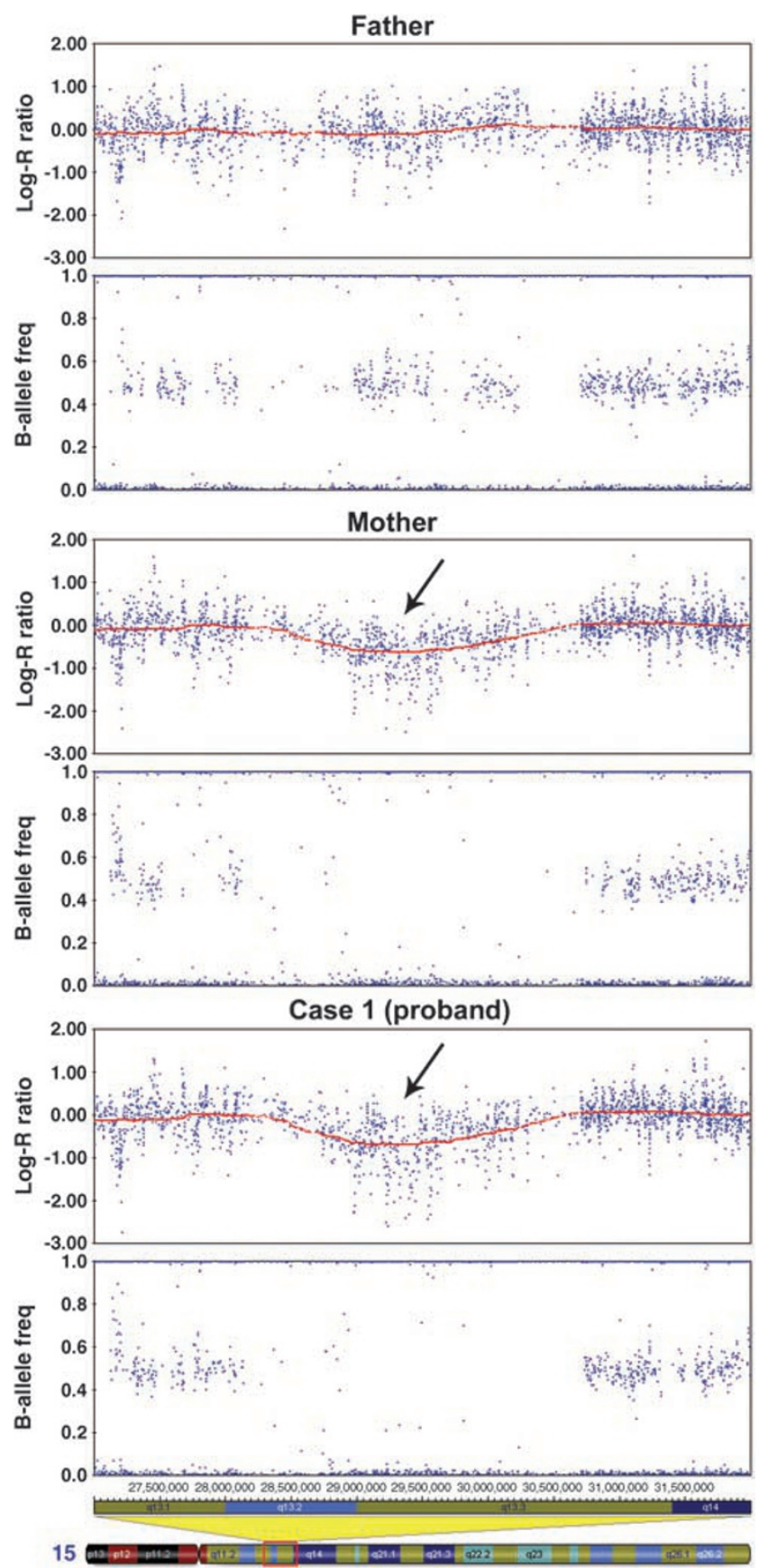

Figure 1 SNP data showing a microdeletion of 15q13.3. $\log R$ ratios and B-allele frequencies are plotted against chromosome 15 position (b36). Visualised using Illumina Genome Viewer (v3.2.7), within BeadStudio. The red line shows a $1 \mathrm{M}$ moving window average of the $\log R$ ratio. Hemizygosity in the mother and proband is shown by a drop in $\log R$ ratio (arrows) and missing heterozygote SNP calls (Ballele frequency $\sim 0.5$ ).

Haplotype analysis of Affymetrix 10K SNPs from an earlier AGP study ${ }^{17}$ was carried out using Merlin ${ }^{18}$ to indicate the most likely pattern of gene flow. This showed that the same maternal haplotype was transmitted to the 

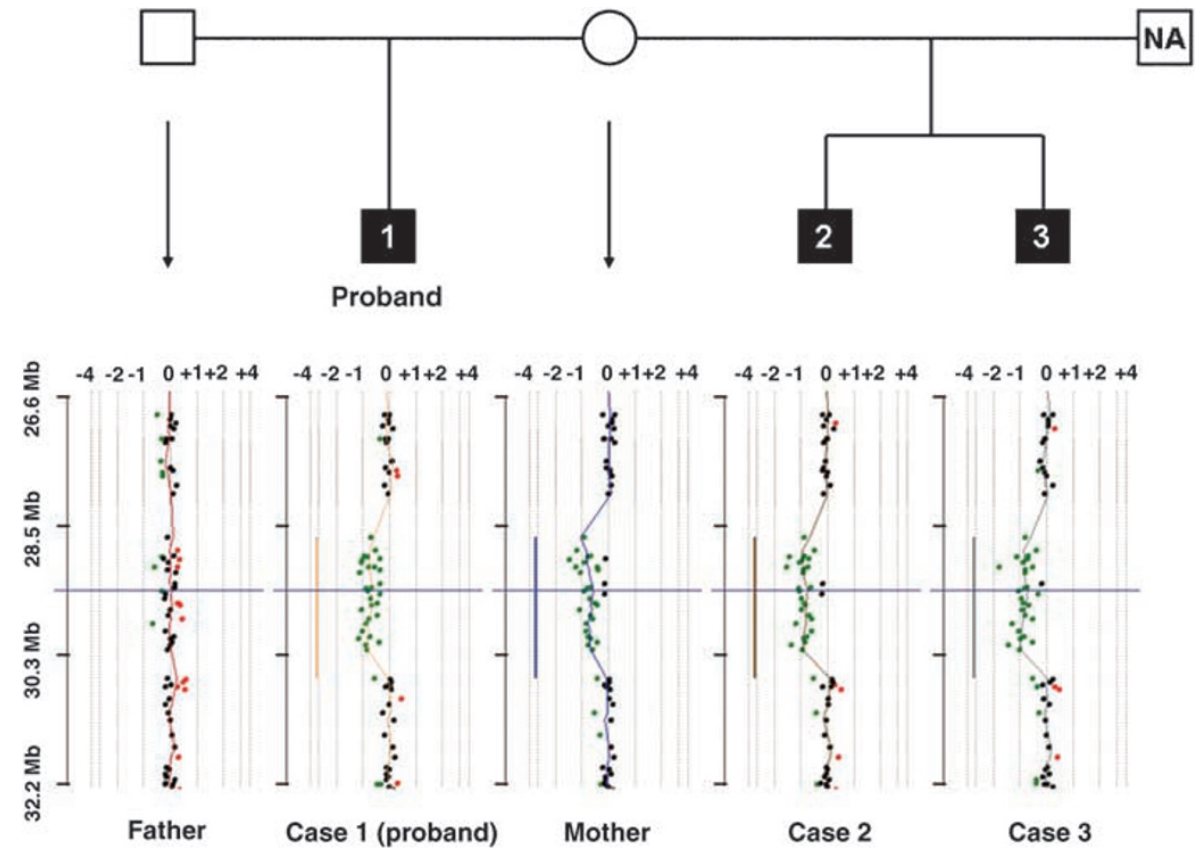

Figure 2 Pedigree and aCGH data shown on CGH Analytics software (v3.4, Agilent). The green and red data points indicate probes with fluorescence ratios falling outside the software cutoff threshold of 0.25 . The deleted regions are indicated by the vertical lines coloured according to the sample, analysed using a moving window average of $0.5 \mathrm{Mb}$. The plots are shown centred on probe A_14_P118326 (chr15: 2948261429482671 , b36 genome coordinates) shown by horizontal blue lines. The scale on the horizontal axes indicates relative copy number change. NA, DNA from this individual was not available.

two affected half-siblings in this region on 15q13 (data not shown). This was confirmed using aCGH, thus demonstrating the segregation of the 15q13.3 microdeletion with autism in this generation of the pedigree (Figure 2). Although there was evidence of learning disabilities in a member of the mother's extended family, further segregation analysis was not possible as DNA was not available.

This family was the only occurrence of the $15 \mathrm{q} 13.3$ microdeletion within the initial AGP cohort (525 probands and their parents, with a multiplex-to-singleton ratio of 2:1 and male-to-female ratio of 5:1). The microdeletion was also not detected in 427 probands from a Canadian autism cohort using Affymetrix $500 \mathrm{~K}$ arrays ${ }^{6}$. No microdeletions of this region have been reported in the Database of Genomic Variants (DGV, http://projects.tcag.ca/variation/; database version of June 2008), ${ }^{19}$ suggesting that the deletion is likely to be of clinical relevance (consistent with the reported MR cases). Conversely, a gain spanning this locus was found in two controls (chr15: $28393128-$ $30740356)^{20}$ and an inversion of this region was detected in a HapMap sample. ${ }^{21}$ Sharp et $a l^{8}$ also detected inversion of this region on 7/16 HapMap chromosomes.

\section{Discussion}

This multiplex family was one of the first to be collected as part of the IMGSAC cohort used for linkage analysis. ${ }^{13,14}$ In this family, the proband and his two maternal half-brothers have all been diagnosed with autism. This family structure (Figure 2) means that recessive susceptibility factors are less likely to be involved in this pedigree, with the most likely inheritance pattern being a maternal dominant risk factor. Consistent with this suggestion, whole-genome CNV analysis identified a 15q13.3 microdeletion that was transmitted to all affected children from their mother. The absence of this deletion from the DGV, together with the segregation seen in this generation of the pedigree, suggests that it may be a rare variant involved in autism pathogenesis. In view of the gender bias seen for autism, it is interesting to note that all the affected offspring are male, whereas the mother harbouring the same deletion appears phenotypically normal.

This 15q13.3 microdeletion was described recently in association with $\mathrm{MR}^{8}$ The nine published MR cases harbouring the microdeletion were described as having mild-to-moderate MR, with reported IQs ranging from 34 to 62 , with one individual having 'mild autism'. By contrast, the three individuals described here are less severely affected in terms of intelligence, but all have been diagnosed with autism using the ADI-R/ADOS. Sharp et $a l^{8}$ also report that the majority of individuals in their study presented with mild face and hand dysmorphologies, whereas no such abnormalities were documented in the family described here. Autism is associated with increased head circumference in approximately $17 \%$ of cases. ${ }^{22}$ Two of the three individuals described here had a head 
circumference of $>97$ th centile, whereas only one of the individuals reported by Sharp et $a l^{8}$ showed this feature (Table 1).

The mechanism by which this deletion might lead to autism can only be speculated upon. Haploinsufficiency of one or more of the seven genes contained in this critical region is likely to be a key factor. One of the genes (CHRNA7) encodes a synaptic ion channel that in mouse knockouts is associated with an abnormal EEG. ${ }^{23}$ This gene is therefore a good candidate to explain the epilepsy or abnormal EEG results observed in seven out of nine of the Sharp et $\mathrm{al}^{8}$ cases (although this phenotype was not present in our study). Alternatively, the microdeletion may also cause changes in chromatin structure that upregulate nearby genes, thereby following the same underlying mechanism as the 15q11-13 duplication.

None of the individuals described here had other associated psychiatric problems at the age of assessment (16 years for case 1, 14 years for case 2 and 10 years for case $3)$. Nevertheless, two recent studies reported the same deletion to be recurrent in schizophrenia cohorts. ${ }^{24,25}$ In the larger of these studies, an Icelandic group found this deletion in 7 out of $4213(0.17 \%)$ affected individuals, compared with 8 out of $39800(0.02 \%)$ controls. Interestingly, 299 of the controls screened were in fact autistic, and one of these autistic control samples harboured the $15 q 13.3$ microdeletion. $^{24}$ In addition, while this paper was under review, Miller et $a l^{26}$ reported this microdeletion in two cases of pervasive developmental disorder - not otherwise specified - from a total of 262 autism spectrum disorder (ASD) and PDD cases referred to Children's Hospital Boston and in 0 out of 751 families from the Autism Genetic Resource Exchange. Together with the individual with milder autism described by Sharp et al ${ }^{8}$ and the autistic 'control', our family is therefore the forth independent report of this rare microdeletion in individuals with ASD and the first to demonstrate segregation in a multiplex family with narrowly defined autism.

It is becoming increasingly apparent that CNVs can span the boundaries of clinical diagnosis. We believe that the $15 q 13.3$ deletion acts in concert with modifier genes, other contributing CNVs or even environmental exposures, which may determine why it causes MR in some individuals, autism in others and schizophrenia in yet more.

Although this microdeletion is rare, its association with autism susceptibility should be further assessed. Studies have shown that the occurrence of de novo CNVs is decreased in multiplex families compared with singleton families; ${ }^{6,27,28}$ so it is therefore important to screen increased numbers of singleton families for this rare variant.

\section{Acknowledgements}

This paper is dedicated to the memory of Professor Annemarie Poustka, who was the founder and head of the Division of Molecular
Genome Analysis at the DKFZ. She was an inspiring scientist and a wonderful person.

We thank Dalila Pinto and the Autism Genome Project (AGP) for CNV data from the initial cohort. The main funders of the AGP are Autism Speaks (USA), Genome Canada (Canada), the Health Research Board (HRB; Ireland), the Hilibrand Foundation (USA), the Medical Research Council (UK) and the National Institute of Health (NIH, USA). We also thank Ghazala Mirza (Genomics laboratory, WTCHG) for performing the Illumina Infinium genotyping, Ernesto Lowy (Bioinformatics, WTCHG) for help with computational data analysis and Janine Lamb for critical comments on the paper. Local funding was from the Nancy Laurie Marks Family Foundation, Simons Foundation, the Wellcome Trust and Deutsche Forschungsgemeinschaft. ESA and SILK were supported by the Oxford Partnership Comprehensive Biomedical Research Centre with funding from the Department of Health's NIHR Biomedical Research Centres funding scheme; the views expressed in this publication are those of the authors and not necessarily those of the Department of Health.

\section{References}

1 Bailey A, Le Couteur A, Gottesman I et al: Autism as a strongly genetic disorder: evidence from a British twin study. Psychol Med 1995; 25 : 63-77.

2 Steyaert JG, De La Marche W: What's new in autism? Eur J Pediatr 2008; 167: 1091-1101.

3 Sykes NH, Lamb JA: Autism: the quest for the genes. Expert Rev Mol Med 2007; 9: 1-15.

4 Baker P, Piven J, Schwartz S, Patil S: Brief report: duplication of chromosome 15q11-13 in two individuals with autistic disorder. J Autism Dev Disord 1994; 24: 529-535.

5 Cook Jr EH, Lindgren V, Leventhal BL et al: Autism or atypical autism in maternally but not paternally derived proximal $15 \mathrm{q}$ duplication. Am J Hum Genet 1997; 60: 928-934.

6 Marshall CR, Noor A, Vincent JB et al: Structural variation of chromosomes in autism spectrum disorder. Am J Hum Genet 2008; 82: $477-488$.

7 Weiss LA, Shen Y, Korn JM et al: Association between microdeletion and microduplication at 16p11.2 and autism. N Engl J Med 2008; 358: 667-675.

8 Sharp AJ, Mefford HC, Li K et al: A recurrent 15q13.3 microdeletion syndrome associated with mental retardation and seizures. Nat Genet 2008; 40: 322-328.

9 Fombonne E: The changing epidemiology of autism. J Appl Res Intellect Disabil 2005; 18: 281-294.

10 Clifford S, Dissanayake C, Bui QM, Huggins R, Taylor AK, Loesch DZ: Autism spectrum phenotype in males and females with fragile X full mutation and premutation. J Autism Dev Disord 2007; 37: 738-747.

11 Ghebranious N, Giampietro PF, Wesbrook FP, Rezkalla SH: A novel microdeletion at $16 \mathrm{p} 11.2$ harbors candidate genes for aortic valve development, seizure disorder, and mild mental retardation. Am J Med Genet A 2007; 143A: 1462-1471.

12 Englert E, Poustka F: [The Frankfurt child and adolescent psychiatry documentation system - development and methodological principles with reference to quality assurance]. Prax Kinderpsychol Kinderpsychiatr 1995; 44: 158-167.

13 International Molecular Genetic Study of Austim Consortium. A full genomewide screen for autism with evidence for linkage to a region on chromosome 7q. Hum Mol Genet 1998; 7: 571-578.

14 International Molecular Genetic Study of Autism Consortium. A genomewide screen for autism: strong evidence for linkage to chromosomes 2q, 7q, and 16p. Am J Hum Genet 2001; 69: 570-581.

15 Colella S, Yau C, Taylor JM et al: QuantiSNP: an Objective Bayes Hidden-Markov Model to detect and accurately map copy number variation using SNP genotyping data. Nucleic Acids Res 2007; 35: 2013-2025. 
16 Pugh TJ, Delaney AD, Farnoud N et al: Impact of whole genome amplification on analysis of copy number variants. Nucleic Acids Res 2008; 36: e80.

17 Szatmari P, Paterson AD, Zwaigenbaum L et al: Mapping autism risk loci using genetic linkage and chromosomal rearrangements. Nat Genet 2007; 39: 319-328.

18 Abecasis GR, Cherny SS, Cookson WO, Cardon LR: Merlin - rapid analysis of dense genetic maps using sparse gene flow trees. Nat Genet 2002; 30: 97-101.

19 Iafrate AJ, Feuk L, Rivera $\mathrm{MN}$ et al: Detection of large-scale variation in the human genome. Nat Genet 2004; 36: 949-951.

20 Pinto D, Marshall C, Feuk L, Scherer SW: Copy-number variation in control population cohorts. Hum Mol Genet 2007; 16 (Spec No. 2): R168-R173.

21 Kidd JM, Cooper GM, Donahue WF et al: Mapping and sequencing of structural variation from eight human genomes. Nature 2008; 453: 56-64.

22 Fombonne E, Roge B, Claverie J, Courty S, Fremolle J: Microcephaly and macrocephaly in autism. J Autism Dev Disord 1999; 29: 113-119.
23 Orr-Urtreger A, Goldner FM, Saeki M et al: Mice deficient in the alpha7 neuronal nicotinic acetylcholine receptor lack alphabungarotoxin binding sites and hippocampal fast nicotinic currents. J Neurosci 1997; 17: 9165-9171.

24 Stefansson H, Rujescu D, Cichon S et al: Large recurrent microdeletions associated with schizophrenia. Nature 2008; 455 : 232-236.

25 Stone JL, O’Donovan MC, Gurling $\mathrm{H}$ et al: Rare chromosomal deletions and duplications increase risk of schizophrenia. Nature 2008; 455: 237-241.

26 Miller DT, Shen Y, Weiss LA et al: Microdeletion/duplication at 15q13.2q13.3 among individuals with features of autism and other neuropsychiatric disorders. J Med Genet 2008. (In press) (doi:10.1136/jmg.2008.059907).

27 Sebat J, Lakshmi B, Malhotra D et al: Strong association of de novo copy number mutations with autism. Science 2007; 316: 445-449.

28 Morrow EM, Yoo SY, Flavell SW et al: Identifying autism loci and genes by tracing recent shared ancestry. Science 2008; 321: $218-223$. 